\title{
Reologia de suspensões de precursor vitrocerâmico do sistema $\mathrm{LiO}_{2}-\mathrm{ZrO}_{2}-\mathrm{SiO}_{2}-\mathrm{Al}_{2} \mathrm{O}_{3}$
}

\section{(Rheology of suspensions of a parent glass belonging to the $\mathrm{LiO}_{2}-\mathrm{ZrO}_{2}-\mathrm{SiO}_{2}-\mathrm{Al}_{2} \mathrm{O}_{3}$ glass-ceramic system)}

\author{
F. R. Cesconeto, J. B. Rodrigues Neto, S. Arcaro, F. Raupp-Pereira, D. Hotza, A. P. Novaes Oliveira \\ ${ }^{1}$ Programa de Pós-Graduação em Ciência e Engenharia de Materiais - PGMAT \\ ${ }^{2}$ Departamento de Engenharia Mecânica - EMC, ${ }^{3}$ Departamento de Engenharia Química - EQA \\ Universidade Federal de Santa Catarina - UFSC, Florianópolis, SC, Brasil 88040-900 \\ franciellycesconeto@gmail.com
}

\begin{abstract}
Resumo
Suspensões cerâmicas em meio aquoso foram preparadas a partir de precursor vitrocerâmico (pó vítreo) do sistema $\mathrm{LZSA}\left(\mathrm{LiO}_{2}{ }^{-}\right.$ $\left.\mathrm{ZrO}_{2}-\mathrm{SiO}_{2}-\mathrm{AlO}_{2}\right)$. As condições de processamento foram estabelecidas através das características do precursor vitrocerâmico e do estudo reológico das suspensões formuladas. A partir da caracterização granulométrica do pó vítreo, variou-se a concentração de sólidos entre 30-40\% (em volume), de dispersante poliacrilato de amônia entre 1,0-3,0\% (PAA) e de ligante polialcoolvinílico (PVA) entre 0,1-0,5\% (com base na massa de sólidos da suspensão). Considerando-se a influência da concentração dos adjuvantes reológicos, assim como as características desejadas para os corpos cerâmicos a verde (elevada densidade), as suspensões foram processadas por colagem de barbotina. Os resultados revelaram que suspensões processadas com $40 \%$ de sólidos, $2 \%$ de PAA e $0,3 \%$ de PVA, proporcionaram as melhores condições para colagem de barbotina do sistema LZSA.
\end{abstract}

Palavras-chave: LZSA, reologia, colagem de barbotina.

\section{Abstract}

Aqueous ceramic suspensions were prepared from a parent glass powder belonging to the $\mathrm{LZSA}_{(}\left(\mathrm{LiO}_{2}-\mathrm{ZrO}_{2}-\mathrm{SiO}_{2}-\mathrm{AlO}_{2}\right)$ glassceramic system. The processing conditions were established by the physical and chemical characteristics of the parent glass powder and by the rheological study of the formulated suspensions. Knowing the characteristics of the glass powder, such as the particle size, the solid content was varied on the suspensions. The solid content was varied from 30 to 40 vol.\% and the additives added were ammonium polyacrylate as dispersant (PAA) and polyvinyl alcohol (PVA) as binder from 1.0 to 3.0\% and 0.1-0.5\% (based on solid weight in the suspension), respectively. Considering the influence of the additives on the rheological behavior as well as the desired characteristics for ceramic green bodies (high density) the suspensions were processed by slip casting. The results showed that suspensions processed with $40 \%$ solids, $2 \%$ PAA, $0.3 \%$ PVA, provided the best conditions for slip casting of LZSA glass powders.

Keywords: glass-ceramics, rheology, slip casting.

\section{INTRODUÇÃO}

Materiais vitrocerâmicos são sólidos policristalinos contendo fase vítrea residual. Vitrocerâmicos do sistema $\mathrm{Li}_{2} \mathrm{O}-\mathrm{ZrO}_{2}-\mathrm{SiO}_{2}-\mathrm{Al}_{2} \mathrm{O}_{3}$ (LZSA) possuem boa resistência química e relativamente baixos coeficientes de expansão térmica $\left(4-6 \times 10^{-6}{ }^{\circ} \mathrm{C}^{-1}\right)$. As principais fases cristalinas formadas durante tratamentos térmicos $\left(700-900{ }^{\circ} \mathrm{C}\right)$ são o espodumênio- $\beta\left(\mathrm{LiAlSi}_{3} \mathrm{O}_{8}\right)$, o silicato de zircônio $\left(\mathrm{ZrSiO}_{4}\right)$ e o metassilicato de lítio $\left(\mathrm{Li}_{2} \mathrm{SiO}_{3}\right)$. Conseqüentemente, os vitrocerâmicos do sistema LZSA têm sido utilizados em aplicações, onde as variações bruscas de temperatura estão presentes como filtros para exaustão de gases quentes ou difusores de chama para secagem a baixas temperaturas $\left(<900{ }^{\circ} \mathrm{C}\right)$. Além disso, estes materiais podem ser obtidos mediante sinterização e cristalização a baixas temperaturas $\left(700-950{ }^{\circ} \mathrm{C}\right)[1]$. Na indústria cerâmica, diversas técnicas de processamento utilizam suspensão de pós cerâmicos em meio aquoso ou orgânico para a conformação de produtos. Dentre as técnicas atrativas e bem estabelecidas de processamento cerâmico que utilizam suspensões para a produção de determinados tipos de componentes destaca-se a colagem de barbotina. Neste tipo de processamento, suspensões estáveis contendo alta fração volumétrica de sólidos e com elevado grau de estabilidade são requisitos importantes para a obtenção de produtos com maior qualidade [2, 3]. Assim, o desenvolvimento de suspensões cerâmicas reologicamente ajustadas é um fator decisivo para a obtenção de materiais com elevada densidade e estabilidade dimensional. Portanto, é fundamental, para uma melhor compreensão dos fatores que afetam a estabilidade de suspensões o conhecimento de aditivos com características físicas e químicas adequadas ao 
precursor vitrocerâmico $[4,5]$.

Neste contexto, este trabalho descreve a preparação e a caracterização reológica de suspensões aquosas de precursor vitrocerâmico do sistema LZSA, buscando a otimização reológica, por meio de supensões com elevada fração volumetrica de sólidos e quantidades adequadas de dispersante (PAA), e plastificante (PVA) para a obtenção de corpos cerâmicos com maiores densidades e boa resistência mecânica a verde.

\section{MATERIAIS E MÉTODOS}

Para realização deste trabalho foi utilizada uma frita do sistema LZSA $\left(16,9 \mathrm{Li}_{2} \mathrm{O} \cdot 5,0 \mathrm{ZrO}_{2} \cdot 65,1 \mathrm{SiO}_{2} \cdot 8,6 \mathrm{Al}_{2} \mathrm{O}_{3}\right)$, preparada a partir de matérias-primas de uso industrial tais como $\mathrm{Li}_{2} \mathrm{CO}_{3}, \mathrm{ZrSiO}_{4}, \mathrm{SiO}_{2}$ e $\mathrm{LiAlSi}_{3} \mathrm{O}_{8}$; poliacrilato de amônia como polieletrólito/dispersante (PAA, Duramax D-3005); ligante poliálcool vinílico (PVA, Vetec) e água destilada $(5 \mu \mathrm{S} / \mathrm{cm}$ e $\mathrm{pH} \sim 7)$. Maiores detalhes sobre a preparação e obtenção da frita LZSA podem ser obtidos no trabalho de Montedo [6]. A frita de LZSA foi colocada em um jarro de porcelana contendo bolas de alumina e moída a seco por $30 \mathrm{~min}$ em moinho rápido (Servitech, CT-242). O pó obtido foi passado em peneira de malha 325 mesh (45 $\mu \mathrm{m})$ e homogeneizado via úmida (proporção entre pó e água de 70/30) em moinho de bolas tipo gira jarro (Cienlab, CE$300 / 120$ ) por $48 \mathrm{~h}$. Subsequentemente, a suspensão foi seca em estufa a $120^{\circ} \mathrm{C}$ por $24 \mathrm{~h}$ e desagregada em moinho de jarro rápido por $5 \mathrm{~min}$. A distribuição de tamanho de partículas foi determinada em um analisador de difração a laser (Malvern, Mastersizer 2000). Concentrações de sólidos de 30, 35 e $40 \%$ em volume foram preparadas e avaliadas pelo modelo matemático de Krieger e Dougherty, pelo qual foi possível definir a fração volumétrica crítica de sólidos [7, 8]. Ainfluência da concentração de dispersante (PAA) e do ligante (PVA) no comportamento (estabilidade) das suspensões foi estudada utilizando-se concentrações de 0 a $3,0 \%$ e de 0,1 a $0,5 \%$ em massa, respectivamente, em relação à concentração de sólidos das suspensões. Todas as suspensões foram homogeneizadas em moinho de jarro rápido com esferas de alumina durante 30 min. Avaliou-se o tempo de homogeneização das suspensões com dispersante por meio de curvas de fluxo obtidas em um viscosímetro (Haake, Polylab System/52p Rheomex). As medidas de fluxo foram realizadas elevando-se a velocidade de cisalhamento de 0 a $1000 \mathrm{~s}^{-1}$ em dois períodos, ascendente e descendente, de $180 \mathrm{~s}$ cada, a uma temperatura constante de $25^{\circ} \mathrm{C}$. O comportamento do ligante foi estudado comparando as viscosidades aparentes e a densidade aparente a verde. As medidas geométricas para calcular a densidade aparente foram feitas utilizando-se um paquímetro (precisão de $\pm 0,01$ $\mathrm{mm}$ ) em corpos-de-prova preparados em moldes cilíndricos de silicone com $10 \mathrm{~mm}$ de altura por $10 \mathrm{~mm}$ de diâmetro. As suspensões foram vertidas em moldes com base porosa em gesso tal que corpos-de-prova foram então desmoldados após $24 \mathrm{~h}$ em temperatura ambiente, secos por $2 \mathrm{~h}$ a $120^{\circ} \mathrm{C}$ em estufa e a massa determinada em balança analítica (precisão $\mathrm{de} \pm 0,001 \mathrm{~g})$.

\section{RESULTADOS E DISCUSSÃO}

O tamanho médio de partícula do precursor vitrocerâmico (LZSA) em água foi $3,8 \mu \mathrm{m}$. Para se avaliar a fração volumétrica crítica de sólidos foi empregado o modelo matemático de Krieger-Dougherty [9, 10], que permite predizer a dependência da viscosidade com a concentração. A Fig. 1 mostra o ajuste dos dados experimentais de acordo com o modelo proposto, e apresenta a variação da viscosidade aparente com a fração volumétrica de sólidos das suspensões aquosas preparadas.

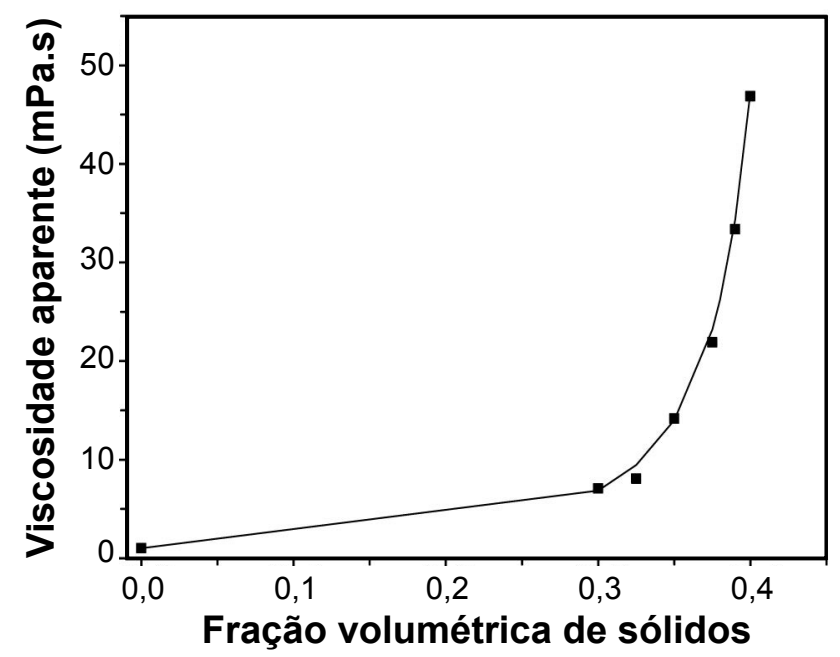

Figure 1: Viscosidade aparente em função da fração volumétrica de sólidos das suspensões.

[Figure 1: Apparent viscosity as a function of volume fraction of solid suspensions.]

A viscosidade aparente aumenta lentamente com a fração volumétrica de sólidos, mas à medida que esta se aproxima da fração máxima, $40 \%$ em volume, passa a crescer rapidamente, tendendo ao infinito. $\mathrm{O}$ comportamento reológico das suspensões do precursor vitrocerâmico (LZSA) sem a adição de dispersante é mostrado na Fig. 2, a qual evidencia um aumento da viscosidade com o aumento da fração de sólidos. Para as concentrações de 30 e $35 \%$ em volume de sólidos a viscosidade é baixa, resultando em suspensões não estáveis e corpos cerâmicos frágeis a verde.

Após obtenção das curvas de fluxo, definiu-se a concentração de sólidos em $40 \%$, como a de viscosidade mais adequada para o processamento por colagem de barbotina. O modelo matemático que melhor descreve o comportamento das suspensões é o modelo de potência (Oswald-de Waele) que descreve um comportamento das curvas de fluxo não linear sem ponto de fluxo [9]. $\mathrm{O}$ parâmetro $\mathrm{n}$, índice de comportamento de fluxo, foi calculado e mostrado na Tabela I.

O parâmetro $\mathrm{n}$ revela que a suspensão com menor carga de sólido ( $30 \%$ em volume) possui um comportamento semelhante ao newtoniano, caracterizado pelo valor de $\mathrm{n}$ próximo de 1 . Pode-se atribuir este fenômeno à baixa concentração de sólidos. Nas suspensões com 35 e $40 \%$ em 


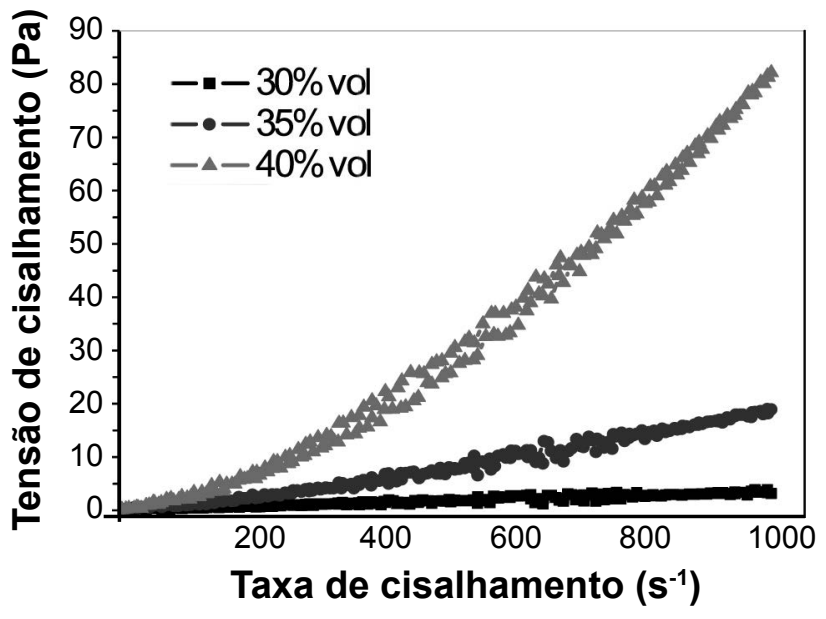

Figura 2: Curvas de fluxo para suspensões de LZSA contendo diferentes concentrações de sólidos.

[Figure 2: Flow curves for LZSA suspensions containing different concentrations of solids.]

Tabela I - Índice de comportamento de fluxo (n) calculado pelo modelo de Oswald-de Waele para as suspensões com o precursor vitrocerâmico LZSA contendo diferentes concentrações de sólidos sem adição de dispersante.

[Table I - Flow behavior index (n) calculated by the Oswaldde Waele model for suspensions with LZSA glass ceramic precursor containing different solids concentrations without addition of a dispersant.]

\begin{tabular}{cc}
\hline Suspensão & $\mathrm{n}$ \\
\hline LZSA 30\% vol de sólido & 0,95 \\
LZSA 35\% vol de sólido & 1,30 \\
LZSA 40\% vol de sólido & 1,60 \\
\hline
\end{tabular}

volume foi observado o comportamento dilatante com $\mathrm{n}>1$, ou seja, a viscosidade aumenta com o aumento da taxa de cisalhamento. Este comportamento dilatante é característico de suspensões com elevados teores de sólidos, onde as partículas encontram-se empacotadas e bastante próximas entre si, tendo, assim, o seu deslocamento dificultado. A Fig. 3 mostra a viscosidade aparente das suspensões cerâmicas (a $500 \mathrm{mPa} . \mathrm{s}$ ), obtida pela razão entre a tensão e a taxa de cisalhamento, em função do teor de dispersante.

Claramente se evidencia (Fig. 3) a diminuição da viscosidade aparente das suspensões de forma acentuada à medida que aumenta a quantidade de PAA até o valor de $2 \%$. Trata-se, provavelmente, de um caso de estabilização do tipo eletrostática, onde o aditivo dispersante é adsorvido em torno das partículas formando uma dupla camada. Assim, as partículas são afastadas uma das outras, diminuindo a força de atração e a desaglomeração das estruturas formadas, diminuindo a viscosidade aparente, resultando em misturas concentradas trabalháveis no intervalo de tempo necessário à sua aplicação [3-5]. O aumento da viscosidade observado para 3\% de dispersante deve-se, provavelmente, ao excesso de PAA que produz reaglomeração das partículas devido à interação do dispersante com a água e interações interfaciais

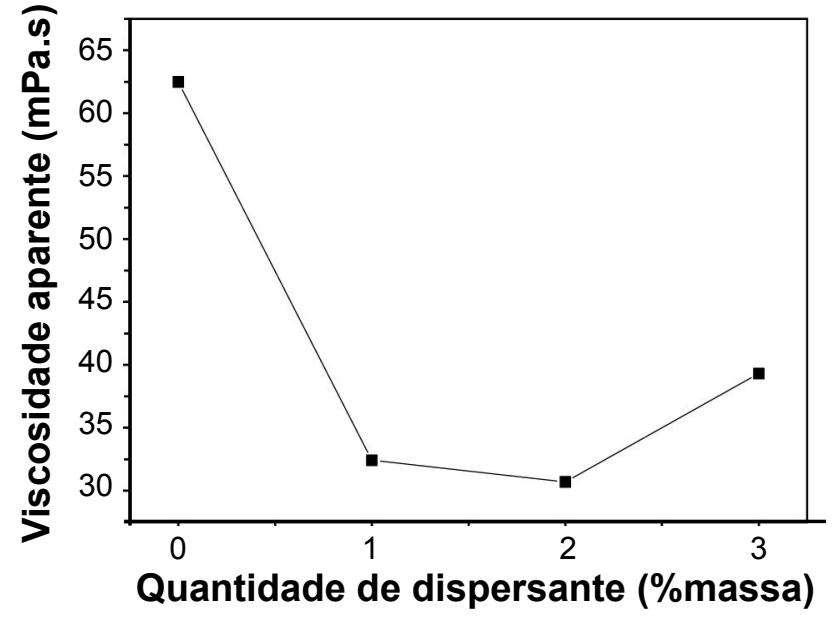

Figura 3: Viscosidade aparente da suspensão com $40 \%$ de sólidos em função da quantidade dispersante.

[Figure 3: Apparent viscosity of the 40\% solids suspension as a function of the dispersant.]

e hidrodinâmicas. As curvas de fluxo, tensão versus taxa de cisalhamento das suspensões cerâmicas contendo $40 \%$ em volume de sólidos com diferentes frações mássicas de dispersante (0, 1, 2, 3\% em massa) são mostradas na Fig. 4.

Observa-se (Fig. 4) que a tensão de cisalhamento diminui com o aumento da fração de dispersante adicionado em relação à suspensão sem aditivo $(0 \%$ PAA), sendo esta redução mais acentuada para a suspensão produzida com $2 \%$ de dispersante (viscosidade menor do que aquelas obtidas com adição de $3 \%$ de PAA). A partir das curvas mostradas na Fig. 4, foi calculado o parâmetro n, conforme o modelo de Oswald-de Waele, Tabela II. Os valores de $\mathrm{n}$ variaram entre 1,30 para a suspensão com $2 \%$ de PAA e 1,60 para suspensões sem dispersante, confirmando o comportamento dilatante, no qual $n>1$.

Nota-se (Tabela II) que o dispersante tem efeito sobre o

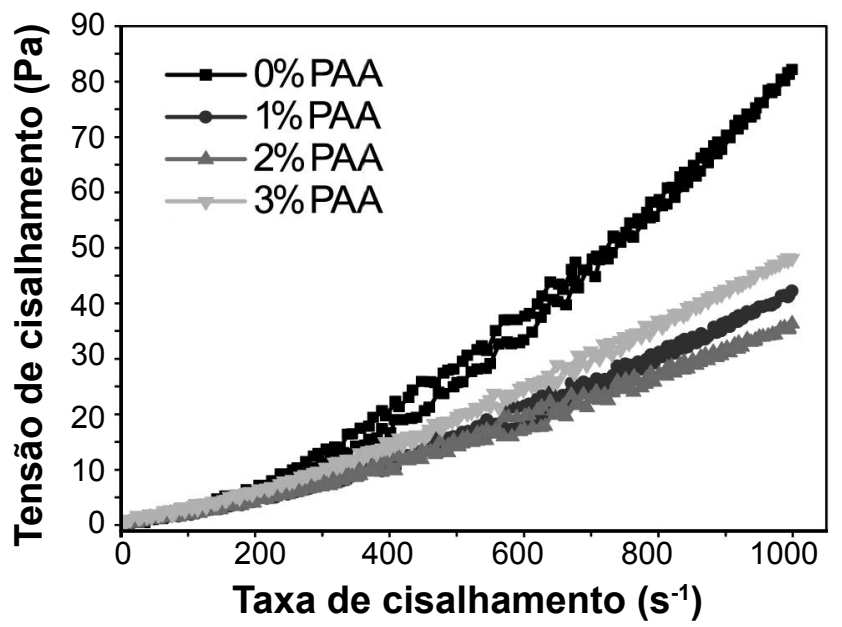

Figura 4: Curvas de fluxo para suspensão com $40 \%$ de precursor vitrocerâmico LZSA contendo diferentes frações mássicas de dispersante (PAA).

[Figure 4: Flow curves for suspension with $40 \%$ glass ceramic precursor LZSA containing different mass fractions of dispersant (PAA).] 
Tabela II - Índice de comportamento de fluxo (n) calculado pelo modelo de Oswald-de Waele para as suspensões cerâmicas preparadas com diferentes concentrações de dispersante (PAA).

[Table II - Flow behavior index (n) calculated by the Oswald-de Waele model for ceramic suspensions prepared with different concentrations of dispersant (PAA).]

\begin{tabular}{cc}
\hline Suspensão com 40\% sólidos & $\mathrm{n}$ \\
\hline LZSA 0\% PAA & 1,60 \\
LZSA 2\% PAA & 1,30 \\
LZSA 3\% PAA & 1,32 \\
\hline
\end{tabular}

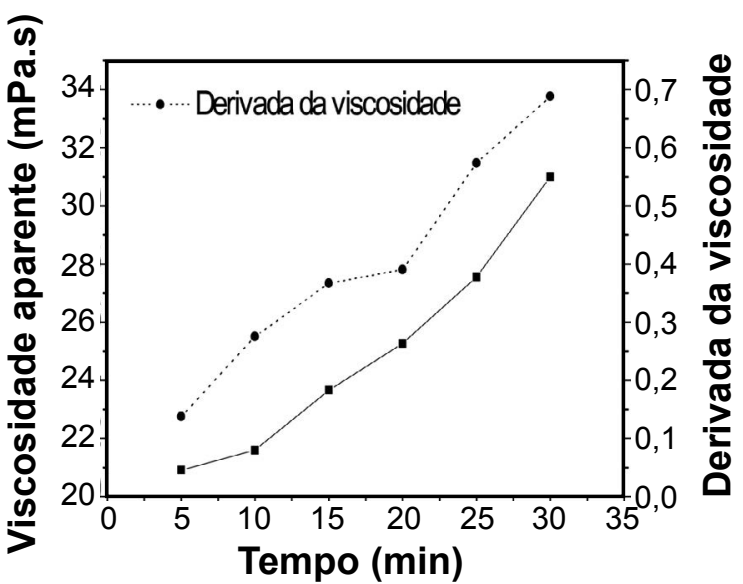

Figura 5: Viscosidade aparente em função do tempo de homogeneização, comparada com a derivada da viscosidade em função do tempo.

[Figure 5: Apparent viscosity as a function of homogenization time, compared to the derivative of viscosity versus time].

comportamento dilatante, observado pelo valor de n, índice de comportamento de fluxo, que diminui com o aumento da concentração de PAA até $2 \%$. A Fig. 5 mostra a variação da viscosidade em função do tempo de homogeneização.

Este comportamento indica o rompimento de possíveis aglomerados de pós muito finos unidos por forças atrativas, que ao serem submetidos a um tempo adequado de homogeneização são destruídos aumentando a quantidade de partículas livres no sistema, conduzindo assim a um aumento na resistência ao fluxo. Ressalta-se que as alíquotas foram retiradas em intervalos pequenos para evitar o fracionamento das partículas e por conseqüência não alterar o teor de dispersante determinado. Ainda na Fig. 5 , para acompanhar a velocidade com que a viscosidade aumenta com o tempo de homogeneização, foi calculada a derivada primeira e pode ser observado um pequeno aumento da viscosidade no intervalo compreendido entre 15 e 20 min de homogeneização, indicando assim o tempo limite. O incremento da viscosidade após o tempo de 20 min de homogeneização está acima do desejado, pois são inadequadas para o processamento proposto neste trabalho. Após determinar o tempo de homogeneização de $15 \mathrm{~min}$, fez-se o estudo da influência do incremento de frações

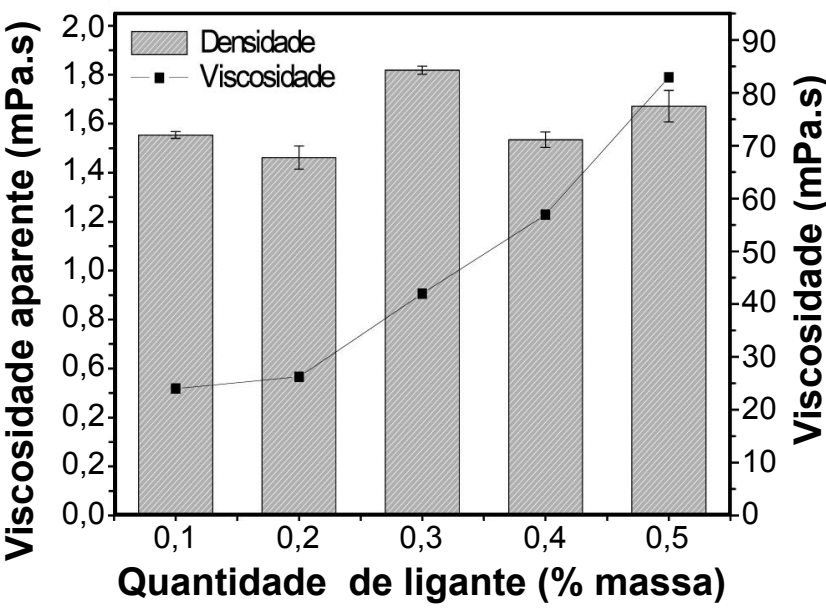

Figure 6: Densidade aparente a verde e variação da viscosidade aparente da suspensão com 40\% de sólidos (LZSA) e 2\% de PAA com a variação do teor de ligante (PVA).

[Figure 6: Raw apparent density and variation of apparent viscosity of the suspension with 40\% solids (LZSA system) and 2\% PAA with varying content of binder (PVA).]

mássicas de ligante (PVA). Este comportamento pode ser observado na Fig. 6 e pode ser atribuído à instauração de pontes orgânicas formadas entre o ligante, a água e as partículas de vidro (precursor vitrocerâmico). Esta interação é responsável pelo aumento da resistência ao fluxo.

A definição da quantidade de ligante foi determinada a partir de medidas de densidade aparente de corpos-de-prova a verde, conformados por colagem de barbotina (ver Fig. 6). A composição com $0,3 \%$ em massa de PVA teve o maior valor de densidade. Este comportamento se deve a um bom empacotamento, menor distância média entre as partículas, resultando em maior densidade a verde [7]. As densidades com concentrações de 0,1 e $0,2 \%$ em massa de PVA variam de forma desordenada, pois existem espaços livres entre as partículas que possibilitam arranjos assimétricos originando corpos com volumes maiores. Devido ao excesso de ligante $(0,4$ e $0,5 \%)$ houve interação e assim formação de aglomerados orgânicos que distanciam as partículas de pó (arranjos assimétricos) e aumentam o volume final do corpo verde originando menores densidades.

\section{CONCLUSÃO}

O estudo reológico se mostrou eficiente na preparação de suspensões aquosas de precursor vitrocerâmico do sistema LZSA $\left(16,9 \mathrm{Li}_{2} \mathrm{O} \cdot 5,0 \mathrm{ZrO}_{2} \cdot 65,1 \mathrm{SiO}_{2} \cdot 8,6 \mathrm{Al}_{2} \mathrm{O}_{3}\right)$ para colagem de barbotina em moldes com base porosa de gesso tendo como bom indicativo a alta densidade a verde. As melhores condições reológicas foram obtidas otimizando a fração volumétrica crítica de sólidos em $40 \%$ em volume, de dispersante poliacrilato de amônia de $2 \%$ em massa em relação aos sólidos. O melhor tempo de homogeneização foi de 15 min e a melhor fração mássica de ligante poliálcool vinílico foi de $0,3 \%$, sendo confirmado com a obtenção da maior densidade a verde, próxima de $1,8 \mathrm{~g} . \mathrm{cm}^{-3}$. 


\section{REFERÊNCIAS}

[1] E. Sousa, C. R. Rambo, F. S. Ortega, A. P. N. Oliveira, V. C. Pandolfelli, Cerâmica 55, 334 (2009) 157.

[2] R. Goren, B. Ersoy, C. Ozgur, T. Alp, Ceram. Int. 38 (2012) 679 .

[3] A. K. Nikumbh, P. V. Adhyapak, Particuology (2012) di:10.1016/j.partic.2011.09.007 (2012).

[4] A. L. Castro, V. C. Pandolfelli, Cerâmica 55, 333 (2009) 18.

[5] E. Sousa, C. R. Rambo, D. Hotza, A. P. N. Oliveira, T.
Fey, Ceram. Trans. 193 (2006) 49.

[6] O. R. K. Montedo, F. M. Bertan, R. Picolli, D. Hotza, A. N. Klein, A. P. N. Oliveira, Am. Ceram. Soc. Bull. 87 (2008) 34.

[7] D. Hotza, Cerâmica 43, 283-284 (1997) 159.

[8] E. Sousa, C. B. Silveira, T. Fey, P. Greil, D. Hotza, A. P. N. Oliveira, Adv. Appl. Ceram. 104 (2005) 22.

[9] R. Moreno, "Reología de suspensiones Cerámicas", $1^{\text {a }}$ Ed., Madrid, Espanha (2005).

[10] E. Sousa, M. Dellú, V. C. Pandolfelli, F. S. Ortega, Cerâmica 57, 341 (2011) 38.

(Rec. 26/03/2013, Rev. 22/07/2013, Ac. 22/09/2013) 